\title{
A Fiber-Optic Electrochemilunescence Sensor Placed into Sample Solution
}

\author{
Changzhi Zhao ${ }^{1}$, Songyun $\mathrm{Xu}^{1}$, Zhaohong Ding ${ }^{1}$, Guoliang Zhao ${ }^{1}$ and Naoyoshi Egashira ${ }^{2}$ \\ ${ }^{1}$ Dept. of Chem., School of Chem. Eng., Dalian Univ. of Technol, 158 Zhongshan Rd., Dalian 116012, \\ China \\ ${ }^{2}$ Dept. of Bio-Resource Chem., Hiroshima Prefectural Univ., Shobara-shi, Hiroshima 727-0023, Japan \\ * Author to whom correspondence should be addressed. Tel: +86-411-4707660. Fax: +86-411- \\ 4707207.E-mail: zhaocz@online.ln.cn.
}

Received: 12 September 2001 / Accepted: 16 September 2001 / Published: 21 September 2001

\begin{abstract}
A novel fiber-optic electrochemiluninescence sensor has been developed. It can be conveniently applied by placing into the sample solution in a manner similar to a conventional ion selective electrode, without requiring liquid luminous reagent and a flowing system. The sensor is consisted of an independent probe and a case for exclusion the ambient light, allowing the sample solution to pass through. Within the body of the probe, an optical fiber is utilized to collect and transmit light signal. In the three electrode configuration of the probe, a Pt electrode coated with $\mathrm{Ru}(\mathrm{bpy})_{3}{ }^{2+}$ - modified chitosan/silica gel membrane is used to give the electrolytic potential and provide a selective luminous membrane. The linear responses of the sensor to oxalic acid and amino acid were obtained in the concentration range of $2.0 \times 10^{-4}$ to $1.0 \times 10^{-2} \mathrm{~mol} / \mathrm{dm}^{3}$ with the relative standard deviations of $3.5 \%$ and $5.7 \%$, respectively. The response of the sensor was not less than $80 \%$ of the initial value after the service time was over one month.
\end{abstract}

Keywords: Electrochemilunescence, Fiber-optic sensor, Modified electrode

\section{Introduction}

Electrochemiluminescence (ECL) has found wide applications in chemistry and biochemistry because of its high sensitivity, relatively simplicity and feasibility under mild conditions [1,2]. Now ECL has not only been used as a detection method for either flow injection analysis or liquid 
chromatography, but also developed into special chemical sensors and biosensors. Egashira et al. fabricated a fiber-optic sensor based on ECL for detecting oxalate [3]. Yokoyama et al. used ECL biosensor in determination of NADH and ethanol [4]. Xu and Bard developed a DNA ECL sensor and applied it in analysis of the hybridization of nucleic acids [5]. Nieman et al. prepared an ECL sensor installed on the photomultiplier tube and determined glucose, and dansyl amino acids [6,7]. Recently, we have prepared an ECL-optical sensor having a Pt electrode coated with $\mathrm{Ru}(\mathrm{bpy})_{3}{ }^{2+}$-modified chitosan and thus successfully improved its selectivity toward oxalic acid [8]. However, these ECL sensors were usually utilized in a flowing system or an electrolytic cell with a dark box for exclusion of ambient light interference from the analytical signal. This configuration is not only inconvenient in operation, but also results in large consumption of luminous reagent. On the basis of our previous work [8], we have developed a fiber-optic ECL sensor that requires neither a flowing system with a dark box, nor liquid luminous reagent. This sensor is a small device that could be dipped into a sample solution in a manner similar to the conventional ion selective electrode. The design of the sensor enables the sample to flow throughout the probe of the sensor so as to ensure sufficient contact of the analyte with the working electrode surface, on which luminous reagent was immobilized. The probe of the sensor contains a three-electrode system that can be changed conveniently so that various working electrodes may be adapted to appropriate analyte. Moreover, by coating an additional protection layer of silica gel on the surface of the Ru(bpy) ${ }_{3}{ }^{2+}$-modified chitosan membrane of the working electrode, durability of the sensor is thus significantly enhanced.

\section{Experimental}

\section{Fabrication of sensor}

The fiber-optic sensor (Figure 1) was consisted of an ECL probe, an internal electrolytic cell and the case of the sensor. The sensor case was made from a dark Teflon rod of $20 \mathrm{~mm}$ in diameter and 30 $\mathrm{mm}$ in length, in the bottom and side of which there were channels that allow the sample solution to flow in and out. In the top of the case, there was a hole of $10 \mathrm{~mm}$ in diameter and $20 \mathrm{~mm}$ in length with thread of $5.0 \mathrm{~mm}$ in depth. An internal electrolytic cell made of PVC tube with bottom was positioned under the thread. From 4 holes on the wall of the electrolytic cell, sample solution could flow throughout the surface of the working electrode. The configuration of the ECL probe was as shown in Figure 2, A. A plastic optical fiber bundle of $2.0 \mathrm{~mm}$ in diameter and $100 \mathrm{~mm}$ in length (Beijing Optoelectrics Co., China) was mounted in a dark Teflon rod $(\phi 5.0 \mathrm{~mm})$, which was surrounded by a Pt wire counter electrode $(\phi 0.8 \mathrm{~mm})$, an $\mathrm{Ag} / \mathrm{AgCl}$ reference electrode $(\phi 0.5 \mathrm{~mm})$, and the working electrode. The working electrode was placed against the end face of the optical fiber in 3$\mathrm{mm}$ space. The ECL probe was inlaid in a dark Teflon rod of $10 \mathrm{~mm}$ in diameter and $50 \mathrm{~mm}$ in length, and fixed into the hole on the top of the sensor with the aid of the screw.

\section{Preparation of working electrode}

To a $75 \mu \mathrm{L}$ tetramethoxysilane (TMOS, Shin-etsu Chem. Co) solution in a mixture of water (1.0 $\mathrm{mL})$ and methanol $(0.4 \mathrm{~mL})$ were added a drop of $0.5 \mathrm{~mol} / \mathrm{dm}^{3}$ hydrochloric acid $(5 \mu \mathrm{L})$ and a drop of 
$2 \%$ ethanol solution of Triton $100(5 \mu \mathrm{L})$. After the sol-gel precursor solution was stirred for $30 \mathrm{~min}$, a drop $(5 \mu \mathrm{L})$ of the precursor solution was applied on an electrode coated with $\mathrm{Ru}(\mathrm{bpy}){ }_{3}{ }^{2+}$-modified chitosan [8] and was dried for $6 \mathrm{~h}$ at room temperature. The same procedure was repeated again to give a working electrode (Figure 2, B) coated with $\mathrm{Ru}(\mathrm{bpy})_{3}{ }^{2+}$-modified chitosan /silica gel membrane of about $6 \mu \mathrm{m}$ in thickness.

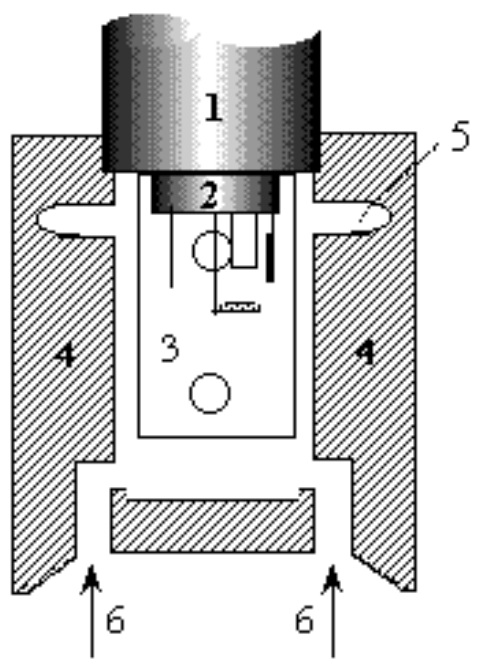

A

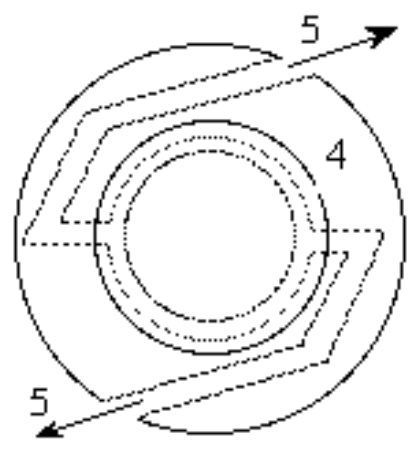

$\mathrm{B}$

Figure 1. Schematic of ECL sensor: A. side views of the sensor; B. top view of the sensor. 1. fixed stick filled with a sensor probe; 2. sensor probe; 3. internal electrolytic cell; 4. sensor body; 5 . outlet of a sample solution; 6 . inlet of a sample solution.
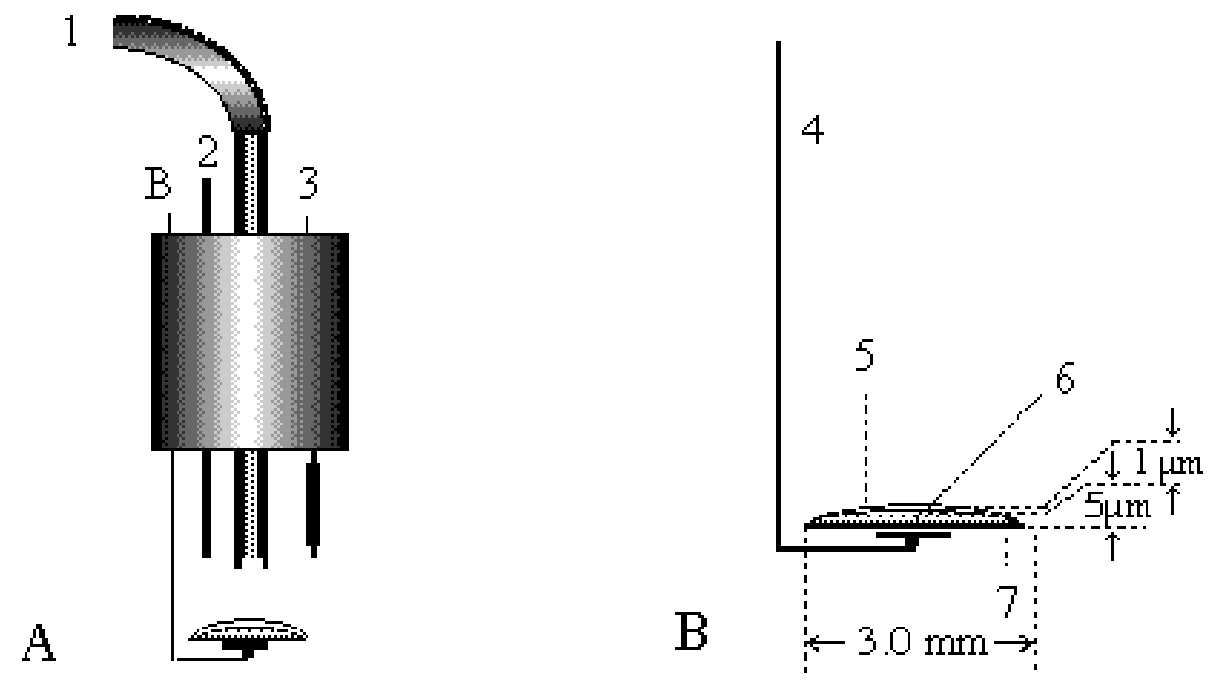

Figure 2. Configuration profile of the sensor probe (A) and Pt electrode coated with chitosan encapsulated Ru (II) complex/silica gel membrane (B). 1. optical fiber; 2. $\mathrm{Ag} / \mathrm{AgCl}$ electrode; 3. Pt counter electrode; 4. Pt wire; 5. Ru (II) complex-modified chitosan layer; 6. silica gel layer; 7. Pt plat. 


\section{Apparatus and procedures}

Votammetric experiments were performed using a CHI660A electrochemical system $(\mathrm{CH}$ Instruments, USA). The standard three-electrode arrangement was consisted of a coated Pt disk $(\phi 1.6$ $\mathrm{mm})$, a Pt counter electrode $(\phi 0.8 \mathrm{~mm})$ and an $\mathrm{Ag} / \mathrm{AgCl}$ reference electrode $(\phi 0.5 \mathrm{~mm})$. A solution containing $0.1 \mathrm{~mol} / \mathrm{dm}^{3} \mathrm{NaNO}_{3}$ and $0.05 \mathrm{~mol} / \mathrm{dm}^{3}$ phosphate buffer was used as the supporting electrolyte. Highly pure nitrogen was passed through the electrolytic cell kept at $25 \pm 0.2$ prior to measurement.

ECL experiments were carried out using the same electrochemical system, the ECL sensor and a DH-1 Fiber-Optic photometer (Shanghai Analytical Instruments, China) at room temperature. Simple ECL determinations, as compared with the general potentiometry, were executed as follows. A 200$\mathrm{mL}$ beaker was employed as the external electrolytic cell, in which a volume amount of supporting electrolyte and the analyte was placed. The sensor was then dipped into the sample solution with steady stirring. The operated voltage of the Fiber-Optic photometer was set at $700 \mathrm{~V}$. After appropriate potential was applied on the sensor, the ECL signal was recorded. The eluting amount of $\mathrm{Ru}(\mathrm{bpy})_{3}{ }^{2+}$ from the membrane was determined by Unicam 949 atomic absorption spectrophotometer equipped with a graphitic furnace (Thermo Instrument Systems, Inc., USA).

\section{Results and Discussion}

\section{Effect of stirring on ECL}

In our newly designed sensor, the electrodes are confined in a small case, and stirring of the tested solution can only be applied outside the cover of the sensor. So we investigate the effect of stirring on ECL at the first place. We have found that stirring in the external electrolytic cell significantly affects the response profile of ECL signal and the response time of the sensor.

As shown in Figure 3, in the case without stirring, the sensor output achieves a maximum within an extremely short time, followed by a non-exponential decay. The signal peak damps to a nearly steady state after $80 \mathrm{~s}$. This result can be explained by the following consideration. For the case without stirring, initially the concentration of the analyte in the vicinity of the electrode surface is as the same of the bulk sample solution, but with the consumption of the analyte during the chemiluminescence (CL) reaction, it will decrease with increasing reaction time. When the steady state is reached, the actual surface concentration is mainly dependent on the mass transfer process of the analyte. Thus, the output profile is characterized by a sharp increase in signal upon imposition of appropriate electrode potential, followed by a non-exponential decay, which typically reflects the mass transfer mode of diffusion control. Since the ECL intensity is proportional to the current passing across the surface of the working electrode, the shape of the output profile and the time for reaching to the steady state are then restricted by the diffusion process.

Contrarily, as stirring is adopted, ECL signal is generated as a single peak and decays rapidly to a steady state within $20 \mathrm{~s}$, and the response profile is similar to those for unconfined sensor under stirring [8]. This implies that when stirring is applied outside the sensor case, the solution in the confined volume can be effectively agitated by the flowing sample solution of the outside part through the small 
holes on the wall of the sensor case. The response profile obtained obviously indicates that in the case with stirring, convection becomes the dominant mode of mass transfer after the earliest moment of ECL. Thus, the output profile will have a smaller increase in signal, but will rapidly decay to the steady state that is significantly higher than obtained in the case without stirring. This steady state level is determined by the analyte concentration in the sample solution and can be used for its quantitation. The ECL responses shown in Figure 4 were collected from the experiments with stirring.

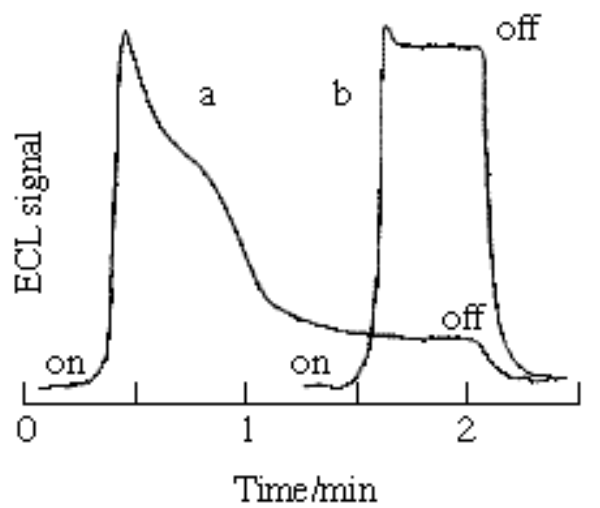

Figure 3. The ECL response of the sensor to oxalic acid without stirring (a) and with stirring (b).

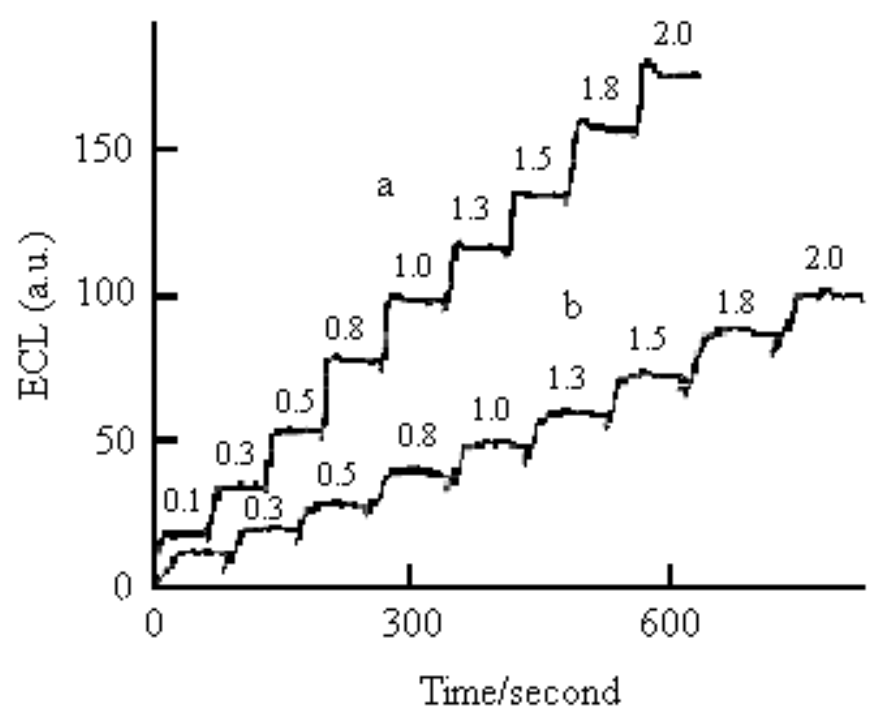

Figure 4. The ECL response of the sensor to oxalic acid (a) and proline (b). The numerals is the concentration $\left(\mathrm{mmol} / \mathrm{dm}^{3}\right)$.

\section{ECL responses of the sensor}

The working electrode we used is similar to that as we have described in our previous work [9], but for improving the longevity, an additional protection layer of silica gel has been coated on the surface of the active layer. For this reason, a test on ECL response of the sensor is necessary.

As shown in Figure 4, the ECL signal for the double layer sensor reaches a steady value within 20 and $30 \mathrm{~s}$ for oxalic acid and proline, and compared with the single layer one, they are 10 and $20 \mathrm{~s}$, respectively. The fact that the response time for proline is longer than for oxalic acid may be due to the slower diffusion of the proline molecule on the chitosan membrane than of the oxalic acid molecule [9]. By using the double layer sensor, the response to concentration gradients of analysts were obtained in the concentration range of $2.0 \times 10^{-4}$ to $1.0 \times 10^{-2} \mathrm{~mol} / \mathrm{dm}^{3}$ (regressive coefficient $\mathrm{r}=0.995$ and 0.992 ) for oxalic acid and proline, and the slopes are $94.6 \mathrm{pA} / \mathrm{mM}$ and $49.5 \mathrm{pA} / \mathrm{mM}$, with the relative standard deviations (RSD) of $3.5 \%$ and $5.7 \%(n=5)$, respectively. For the single layer sensor, however, the corresponding slopes are $100 \mathrm{pA} / \mathrm{mM}$ and $78 \mathrm{pA} / \mathrm{mM}$. 


\section{Durability of the sensor}

The aim of this work is to fabricate a fiber-optic ECL sensor of small size that can be placed into the sample solution and conveniently used in a manner similar to an ion selective electrode. To meet the requirement of practical application, it must have a longer service time and higher repeatability. For this purpose, the procedure for preparation of the working electrode has been improved, compared with our previous work [8]. That is, the modified layer of the working electrode is consists of both membranes of chitosan and silica gel. The first layer is a sensing membrane of chitosan encapsulated $\mathrm{Ru}(\mathrm{bpy})_{3}{ }^{2+}$, which was immobilized on the surface of the electrode with the aid of high viscosity of chitosan. The second layer, silica gel, is a protective membrane coated on the chitosan film could not only effectively restrain the swelling of chitosan, but also inhibits the elution of $\mathrm{Ru}(\mathrm{bpy})_{3}{ }^{2+}$ from the membrane. Figure 5 shows variation of the response to proline within 60 days as contrasted with the eluted amount of $\mathrm{Ru}(\mathrm{bpy})_{3}{ }^{2+}$ from the membrane of the probes with and without silica gel protection, respectively. The response of the doubly coated electrode decreases slightly over one month and more steeply after forty days when it decreases to about $80 \%$ of the initial value, while the response of the single layer one descends abruptly just after one week. These results are consistent with the elution of $\mathrm{Ru}(\mathrm{bpy}){ }_{3}{ }^{2+}$ from the membrane. This can be used to explain why silica gel coating can enhance the durability of the electrode.

The experimental results suggest that although our newly developed sensor has a little longer response time and lower sensitivity compared with that described in our previous work [8], its longevity have been significantly improved by adding a protection layer of silica gel on the active surface of the electrode.

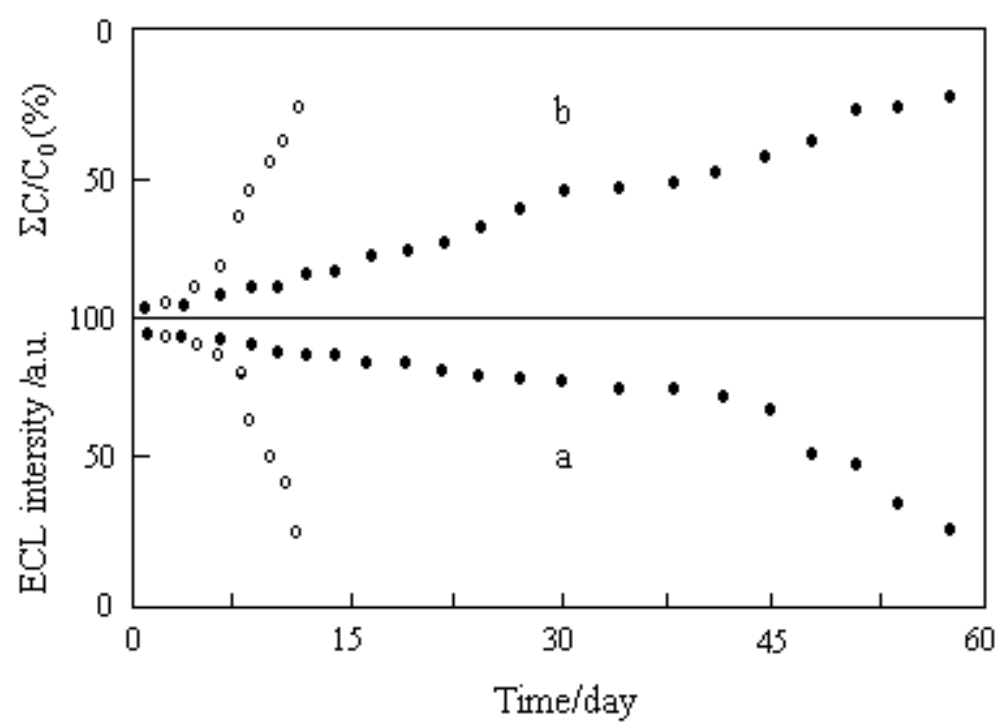

Figure 5. Decays of the responses of the probe prepared with doubly coated electrode $(\bullet)$ and the $\mathrm{Ru}(\mathrm{II})$-modified chitosan electrode (o) to $1.00 \times 10^{-3} \mathrm{~mol} / \mathrm{dm}^{3}$ proline (a). Eluate amount of Ru-complex from the membrane in relation to decrease of the responses (b). 


\section{Acknowledgements}

This project is sponsored by the Scientific Research Foundation for the Returned Overseas Chinese (SRF for ROCS: No: 1998-679), and to which all the authors here are grateful.

\section{References}

1. Rubinstein, I.; Martin, C. R.; Bard, A. J. Electrogenerated chemiluminescence determination of oxalate. Anal. Chem. 1983, 55, 1580-1582.

2. Lee, W. Y. Tris(2,2'-bipyridine) ruthenium Electrogenerated chemiluminescence in analytical science. Michrochim. Acta 1997, 127, 19-39.

3. Egashira, N.; Kumasako, Y.; Ohga, K. Fabrication of a fiber-optic-based electrochemiluminescence sensor and its application to the determination of oxalate, Anal. Sci. 1990, 6, 903-904.

4. Yokoyama, K.; Sasaki, S.; Ikebukuro, K.; Takeuchi, T.; Tokitsu, Y.; Masuda, Y. Biosensing based on NADH detection coupled to electrogenerated chemiluminescence from tris(2,2'-bipyridine) ruthenium, Talata 1994, 41, 1035-1039.

5. $\mathrm{Xu}, \mathrm{X} . \mathrm{H}$;; Bard, A. J. Immobilization and hybridization of DNA on an aluminum (III) alkanebisphosphonate thin film with Electrogenerated chemiluminescence detection.. J. Am. Chem. Soc. 1995, 117, 2627-2631.

6. Martin, A. F.; Nieman, T. A. Glucose quantitation using an immobilized glucose dehydrogenase enzyme reactor and a tris(2,2'-bipyridine) ruthenium(II) chemiluminescent sensor, Anal. Chim. Acta 1993, 281, 475-481.

7. Skotty, D. R.; Lee, W. Y.; Nieman, T. A. Determination of dansyl amino acids and oxalate by HPLC with electrogenerated chemiluminescence determination using tris(2,2'-bipyridine) ruthenium(II) in the mobile phase. Anal. Chem. 1996, 68, 1530-1535.

8. Zhao, C.; Egashira, N.; Kurauchi, Y.; Ohga, K. Electrochemiluminescence Oxalic Acid Sensor Having a Platinum Electrode Coated with Chitosan Modified with a Ruthenium (II) Complex, Electrochim. Acta 1998, 43, 2167-2173.

Sample Availability: Available from the author.

(C) 2001 by MDPI (http://www.mdpi.net). Reproduction is permitted for noncommercial purposes. 